\title{
Diagnostic limitations of magnifying endoscopy with narrow- band imaging in early gastric cancer
}

\section{다 (용}

\begin{abstract}
Authors Nagahara ${ }^{1}$

Institutions

1 Department of Gastroenterology, Juntendo University, School of Medicine, Tokyo, Japan

2 Department of Human Pathology, Juntendo University, School of Medicine, Tokyo, Japan
\end{abstract}

Kohei Matsumoto', Hiroya Ueyama', Takashi Yao², Daiki Abe ${ }^{1}$, Shotaro Oki ${ }^{1}$, Nobuyuki Suzuki ${ }^{1}$, Atsushi Ikeda',

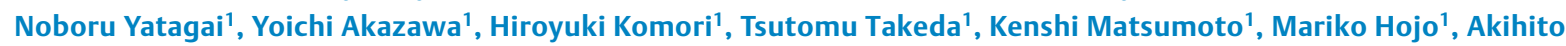

submitted 19.2.2020

accepted after revision 8.5.2020

\section{Bibliography}

Endoscopy International Open 2020; 08: E1233-E1242

DOI 10.1055/a-1220-6389

ISSN 2364-3722

(C) 2020. The Author(s).

This is an open access article published by Thieme under the terms of the Creative Commons Attribution-NonDerivative-NonCommercial License, permitting copying and reproduction so long as the original work is given appropriate credit. Contents may not be used for commecial purposes, or adapted, remixed, transformed or built upon. (https://creativecommons.org/licenses/by-nc-nd/4.0/)

\section{Corresponding author}

Hiroya Ueyama, MD, PhD, Department of Gastroenterology, Juntendo University School of Medicine, 2-1-1 Hongo,

Bunkyo-Ku, Tokyo 113-8421, Japan

Fax: +81-3-3813-8862

psyro@juntendo.ac.jp

\section{ABSTRACT}

Background and study aims Magnifying endoscopy with narrow band imaging ( $\mathrm{M}-\mathrm{NBI}$ ) has made a huge contribution to endoscopic diagnosis of early gastric cancer (EGC). However, we sometimes encountered false-negative cases with M-NBI diagnosis (i.e., M-NBI diagnostic limitation lesion: M-NBI-DLL). However, clinicopathological features of $\mathrm{M}-\mathrm{NBI}$-DLLs have not been well elucidated. We aimed to clarify the clinicopathological features and histological reasons of M-NBI-DLLs.

Patients and methods In this single-center retrospective study, M-NBI-DLLs were extracted from 456 EGCs resected endoscopically at our hospital. We defined histological types of M-NBI-DLLs and analyzed clinicopathologically to clarify histological reasons of M-NBI-DLLs.

Results of 456 EGCs, 48 lesions (10.5\%) of M-NBI-DLLs were enrolled. M-NBI-DLLs was classified into four histological types as follows: gastric adenocarcinoma of fundicgland type (GA-FG, $n=25$ ), gastric adenocarcinoma of fundic-gland mucosal type (GA-FGM, $n=1)$, differentiated adenocarcinoma $(n=14)$, and undifferentiated adenocarcinoma $(n=8)$. Thirty-nine lesions of M-NBI-DLLs were $H$. pylorinegative gastric cancers (39/47, 82.9\%). Histological reasons for M-NBI-DLLs were as follows: 1) completely covered with non-neoplastic mucosa (25/25 GA-FG, 8/8 undifferentiated adenocarcinoma); 2) well-differentiated adenocarcinoma with low-grade atypia (1/1 GA-FGM, 14/14 differentiated adenocarcinoma); 3) similarity of surface structure (10/14 differentiated adenocarcinoma); and 4) partially covered and/or mixed with a non-neoplastic mucosa (1/1 GA-FGM, 6/14 differentiated adenocarcinoma).

Conclusions Diagnostic limitations of $\mathrm{M}-\mathrm{NBI}$ depend on four distinct histological characteristics. For accurate diagnosis of M-NBI-DLLs, it may be necessary to fully understand endoscopic features of these lesions using white light imaging and M-NBI based on these histological characteristics and to take a precise biopsy.

\section{Introduction}

Magnifying endoscopy with narrow band imaging (M-NBI) has made a huge contribution to endoscopic diagnosis of early gastric cancer (EGC) and its use has become commonplace in gastrointestinal endoscopy [1]. Recently, an algorithm for the magnifying endoscopy diagnosis of EGC (MESDA-G: Magnifying
Endoscopy Simple Diagnostic Algorithm for early Gastric cancer) was proposed [2], and the usefulness of $\mathrm{M}-\mathrm{NBI}$ in diagnosing EGC is high [3-13]. However, we have sometimes encountered false-negative cases with $\mathrm{M}-\mathrm{NBI}$ diagnosis (endoscopists diagnosed as non-cancerous with $\mathrm{M}-\mathrm{NBI}$ but the pathological diagnosis was cancerous) (i.e., M-NBI diagnostic limitation lesion: M-NBI-DLL). 


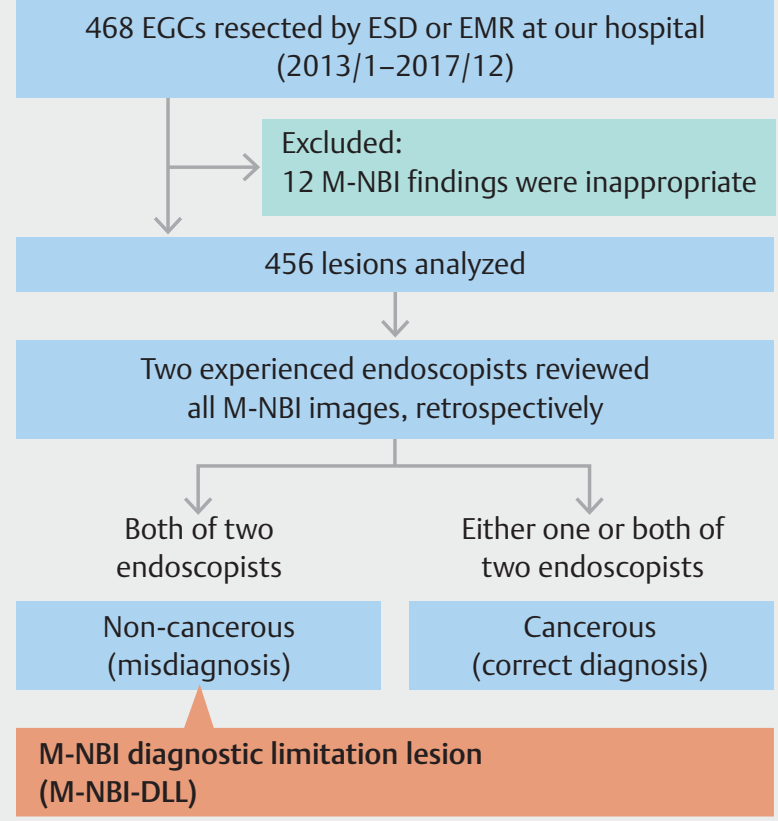

- Fig. 1 Flowchart of case selection and analysis. EGC, early gastric cancer; ESD, endoscopic submucosal dissection; EMR, endoscopic mucosal resection; M-NBI, magnifying endoscopy with narrow band imaging.

In 2010, we proposed classifying differentiated adenocarcinoma with low-grade atypia, especially those presenting with a marked predominance of differentiation into chief cells, as a new type, namely gastric adenocarcinoma of fundic-gland type (GA-FG) [14]. GA-FG is a notable new entity in gastric cancer, which was described as an oxyntic gland adenoma or gastric adenocarcinoma of fundic-gland type in WHO Classification of Tumours, 5th Edition, published in 2019 [15]. Recently, in addition to a fundic-gland type, a few cases of a GA-FG subtype, with a tendency of differentiating toward a foveolar-epithelium type, have been reported as gastric adenocarcinoma of fundic-gland mucosal type (GA-FGM) [16-18].

GA-FG is often diagnosed as non-cancerous by M-NBI, implying a M-NBI diagnosis is limited when applied to such lesions. Most GA-FG appear to be a type of Helicobacter pylori (Hp)-naïve gastric cancer. Histological types of $\mathrm{Hp}$-naïve gastric cancers are generally dominated by undifferentiated adenocarcinoma and differentiated adenocarcinoma with low-grade atypia, including GA-FG [19].

This suggests some M-NBI-DLLs may be included in Hp-naïve gastric cancers. Accompanied by widespread use of $\mathrm{Hp}$ eradication therapy in recent years, the incidence of $\mathrm{Hp}$-naïve gastric cancers has increased. However, clinicopathological features of M-NBI-DLLs have not been well elucidated. We aimed to clarify the clinicopathological features and histological reasons of M-NBI-DLLs and investigate their $\mathrm{Hp}$ infection status.

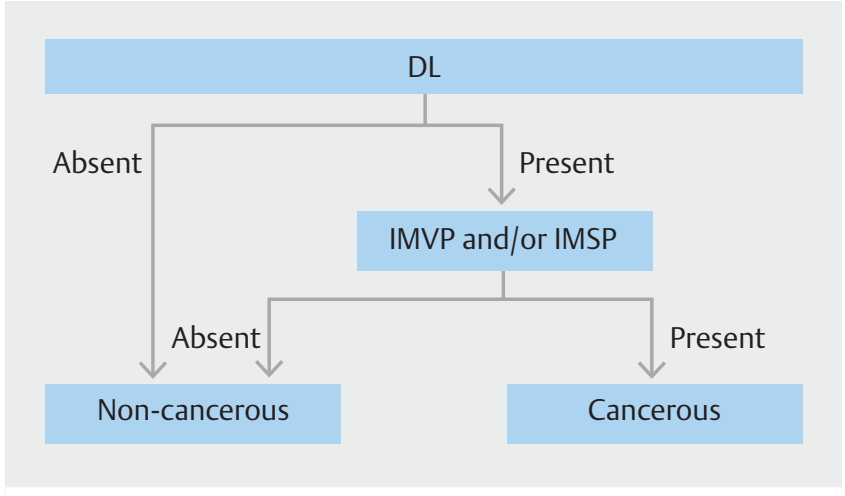

- Fig. 2 Vessel plus surface classification system (VSCS). DL, demarcation line; IMVP, irregular microvascular pattern; IMSP, irregular microsurface pattern.

\section{Patients and methods}

This study was conducted in accordance with the Declaration of Helsinki and approved by the Institutional Review Board of Juntendo University School of Medicine (approval number: JHS 170027). Patients were not required to give consent for the study because the analysis used anonymous clinical data that were obtained after each patient agreed to treatment by verbal and documental consent. Individuals cannot be identified from the data presented.

\section{Study design}

We retrospectively reviewed both $\mathrm{M}-\mathrm{NBI}$ images and resected specimens from a total of 468 EGCs in 439 consecutive patients who had undergone preoperative $\mathrm{M}-\mathrm{NBI}$ examination and endoscopic resection between January 2013 and December 2017 at our hospital ( $>$ Fig. 1). Lesions for which M-NBI images were not taken at maximum magnification or were difficult to evaluate because of mucus or erosions were excluded. After exclusion, a total of 456 lesions in 427 patients were enrolled in this study. Of these 456 lesions, false-negative cases with M$\mathrm{NBI}$ diagnosis (= M-NBI-DLL) were extracted and subjected to a clinicopathological evaluation.

\section{M-NBI diagnostic criteria}

We employed a vessel plus surface classification system (VSCS) [20] which is main algorithm of MESDA-G for an M-NBI diagnosis of EGC ( $\mathbf{F i g . 2 )}$. Two expert endoscopists (H.U. and K.M.) reviewed $\mathrm{M}-\mathrm{NBI}$ images of all cancerous lesions. Both of two endoscopists checked M-NBI images of all lesions whether a lesion complied with the cancerous diagnostic criteria of the VSCS. Lesions that did not fulfill the cancerous diagnostic criteria of VSCS (any patterns of microvascular (MV) and microsurface (MS) without a demarcation line (DL), or regular MV and regular MS pattern with a DL) were defined as M-NBI-DLL, and we excluded misdiagnosed horizontal margin lesions from $\mathrm{M}$ NBI-DLL. 
- Table 1 Histological diagnoses in this study.

\begin{tabular}{|c|c|c|}
\hline $\begin{array}{l}\text { Vienna } \\
\text { Category }\end{array}$ & Diagnosis & \\
\hline 1 & Negative for neoplasia & \multirow{5}{*}{$\begin{array}{l}\text { Non-can- } \\
\text { cerous }\end{array}$} \\
\hline 2 & Indefinite for neoplasia & \\
\hline \multirow[t]{3}{*}{3} & Mucosal low-grade neoplasia & \\
\hline & - Low-grade adenoma & \\
\hline & - Low-grade dysplasia & \\
\hline \multirow[t]{5}{*}{4} & Mucosal high-grade neoplasia & \multirow[t]{6}{*}{ Cancerous } \\
\hline & - 4.1 High-grade adenoma/dysplasia & \\
\hline & $\begin{array}{l}\text { - } 4.2 \text { Non-invasive carcinoma } \\
\text { (carcinoma in situ) }\end{array}$ & \\
\hline & - 4.3 Suspicious for invasive carcinoma & \\
\hline & - 4.4 Intramucosal carcinoma & \\
\hline 5 & Submucosal invasion by carcinoma & \\
\hline
\end{tabular}

\section{Determination of $\mathrm{Hp}$ infection status}

$\mathrm{Hp}$ infection status of M-NBI-DLLs was examined. A status of $\mathrm{Hp}$ positive was defined as positive for at least one of the following current $\mathrm{Hp}$ infection tests: rapid urease test (Otsuka Pharmaceutical Co., Tokyo, Japan), ${ }^{13} \mathrm{C}$-urea breath test (Photal Otsuka Electronics, Tokyo, Japan) and $H p$ stool antigen test (Premier Platinum HpSA; Meridian, Cincinnati, Ohio, United States).

$\mathrm{Hp}$-negative included the statuses of $\mathrm{Hp}$-naive and post- $\mathrm{Hp}$ eradication. A status of $\mathrm{Hp}$-naïve was defined as the fulfillment of all the following criteria: no eradication history, no mucosal atrophy in endoscopic (no atrophy in the stomach or C-1 according to Kimura-Takemoto Classification [21]) and pathological findings, and negative for at least one of the following tests: rapid urease test, ${ }^{13} \mathrm{C}$-urea breath test, $\mathrm{Hp}$ stool antigen test and serum $\mathrm{Hp}$ antibody (Eiken, Tokyo, Japan).

If the patient had a history of $\mathrm{Hp}$ eradication and negative for current $\mathrm{Hp}$ infection test above-mentioned, the patient was diagnosed with post-Hp eradication without current infection. Post-Hp eradication gastric cancer was defined as a lesion diagnosed by endoscopy a year or more after successful eradication.

\section{Histological investigation}

Specimens obtained by endoscopic resection were histologically evaluated. A histological diagnosis was made according to a revised Vienna classification by a highly experienced gastrointestinal pathologist (T.Y.) at our hospital. For the purpose of this study, revised Vienna categories C4 and C5 were reclassified as cancerous, and all other classifications as non-cancerous ( $\sim$ Table 1) [22]. Gastric cancer was histologically typed according to the Japanese Classification of Gastric Carcinoma, 15th edition [23]. Intestinal/ diffuse/indeterminate types of the Lauren classification corresponded to differentiated/undifferentiated/special types according to the Japanese Classification of Gastric Carcinoma 15th edition.
Table 2 Clinicopathological features of 456 early gastric cancers from 427 patients.

\begin{tabular}{|l|c|}
\hline Number of lesions, $\mathrm{n}$ & 456 \\
\hline Male/female & $298 / 129$ \\
\hline Age, years, median (range) & $71.3(35-92)$ \\
\hline Location: upper/middle/lower & $78 / 175 / 203$ \\
\hline Tumor size, mm, mean (range) & $14.9(1-56)$ \\
\hline Macroscopic type & \\
\hline - elevated type & 180 \\
\hline - flat and depressed type & 276 \\
\hline Histological type & \\
\hline - Differentiated type (intestinal type) & \\
\hline - tubular adenocarcinoma & 358 \\
\hline - papillary adenocarcinoma & 7 \\
\hline - Undifferentiated type (diffuse type) & \\
\hline - signet-ring cell carcinoma & 19 \\
\hline - poorly differentiated adenocarcinoma & 5 \\
\hline - & $379 / 77$ \\
\hline - - Mepthed type (differentiated plus undifferen- & 34 \\
\hline - - Gated type) & 25 \\
\hline - GA-FG & 3 \\
\hline - Special type (indeterminate type) & $1.5(50-5000)$ \\
\hline
\end{tabular}

GA-FG, gastric adenocarcinoma of fundic-gland type; GA-FGM, gastric adenocarcinoma of fundic-gland mucosal type; M, mucosa; SM, submucosa

Histological findings were analyzed according to presence or absence of: (1) well-differentiated adenocarcinoma with lowgrade atypia (defined as well-differentiated adenocarcinoma with low-grade cellular and architectural atypia, the judgment of which was hard to make for a diagnosis of cancer or inflammation); (2) similarity of surface structure (defined as few differences between density of neoplastic glandular duct and non-neoplastic glandular duct); (3) partially covered with nonneoplastic mucosa on the tumor surface; and (4) completely covered with non-neoplastic mucosa on the tumor surface. 
- Table3 Clinicopathological and histological features of M-NBI-DLLs.

\begin{tabular}{|c|c|c|c|c|c|}
\hline & GA-FG & GA-FGM & $\begin{array}{l}\text { Differentiated } \\
\text { adenocarcinoma }\end{array}$ & $\begin{array}{l}\text { Undifferentiated } \\
\text { adenocarcinoma }\end{array}$ & Total \\
\hline Number of lesions, $\mathrm{n}$ & 25 & 1 & 14 & 8 & 48 \\
\hline Male/female & $13 / 12$ & $1 / 0$ & $9 / 5$ & $4 / 4$ & $27 / 21$ \\
\hline Age, years, mean (range) & $67.4(51-80)$ & 63 & $70.2(46-81)$ & $55(41-73)$ & $66(41-81)$ \\
\hline Location: upper/middle/lower & $21 / 2 / 2$ & $1 / 0 / 0$ & $0 / 8 / 6$ & $0 / 2 / 6$ & $22 / 12 / 14$ \\
\hline Tumor size, mm, mean (range) & $9(2-43)$ & 15 & $12.6(0.8-35)$ & $6.4(2-12)$ & $9.7(1-43)$ \\
\hline \multicolumn{6}{|l|}{ Macroscopic type } \\
\hline - elevated type & 16 & 1 & 5 & 0 & 22 \\
\hline - flat or depressed type & 9 & 0 & 9 & 8 & 26 \\
\hline Depth of invasion: M/SM & $7 / 18$ & $0 / 1$ & $14 / 0$ & $8 / 0$ & 29/19 \\
\hline Median SM invasive width, $\mu \mathrm{m}$ (range) & $341.7(50-1400)$ & 700 & & & \\
\hline DL: positive/negative & $2 / 23$ & $0 / 1$ & $5 / 9$ & $0 / 8$ & $7 / 41$ \\
\hline MV: regular/irregular/absent & $25 / 0 / 0$ & $1 / 0 / 0$ & $11 / 2 / 1$ & $8 / 0 / 0$ & $45 / 2 / 1$ \\
\hline MS: regular/irregular/absent & $25 / 0 / 0$ & $1 / 0 / 0$ & $12 / 2 / 0$ & $8 / 0 / 0$ & $46 / 2 / 0$ \\
\hline \multicolumn{6}{|l|}{ Histological findings } \\
\hline $\begin{array}{l}\text { - (1) Well-differentiated adenocarcinoma } \\
\text { with low-grade atypia }\end{array}$ & $25 / 25(100 \%)$ & 0 & 0 & $8 / 8(100 \%)$ & \\
\hline - (2) Similarity of surface structure & 0 & $1 / 1(100 \%)$ & $14 / 14(100 \%)$ & 0 & \\
\hline $\begin{array}{l}\text { - (3) Partially covered with a non-neoplastic } \\
\text { mucosa }\end{array}$ & 0 & $1 / 1(100 \%)$ & $6 / 14(42.9 \%)$ & 0 & \\
\hline $\begin{array}{l}\text { - (4) Completely covered with a non- } \\
\text { neoplastic mucosa }\end{array}$ & 0 & 0 & $10 / 14(71.4 \%)$ & 0 & \\
\hline
\end{tabular}

\section{Results}

\section{Clinicopathological findings}

- Table 2 shows the clinicopathological features of 456 EGCs from 427 patients. Of 456 EGCs, 48 lesions (10.5\%) were MNBI-DLLs. Males predominated (M/F, 298/129), with an average age of 71.3 years (range 35-92). Tumor locations were lower third $(\mathrm{L})>$ middle third $(\mathrm{M})>$ upper third $(\mathrm{U})=203 / 175 / 78$, respectively. Mean tumor diameter was $14.9 \mathrm{~mm}$ (range 156). For macroscopic type, flatter and more depressed than elevated types were found (flat and depressed/elevated, 276/ 180). The histological types were as follows: 358 tubular adenocarcinomas and seven papillary adenocarcinomas among differentiated adenocarcinomas, 19 signet-ring cell carcinomas and five poorly differentiated adenocarcinomas among undifferentiated adenocarcinomas, 34 mixed type (differentiated plus undifferentiated) adenocarcinomas, 25 GA-FG, and 3 GAFGM. There were three gastric adenocarcinomas with enteroblastic differentiation, one hepatoid adenocarcinoma, and one gastric carcinoma with lymphoid stroma among special type gastric carcinomas. In terms of tumor depths, more tumors showed mucosal than submucosal invasion (mucosal/submucosal, 379/77). Mean depth of submucosal invasion was $731.5 \mu \mathrm{m}$ (range 50-5000).

- Table 3 shows the clinicopathological features of M-NBIDLLs. M-NBI-DLLs was classified into four histological types as follows; GA-FG $(n=25)$, GA-FGM $(n=1)$, differentiated adenocarcinoma $(n=14)$, and undifferentiated adenocarcinoma $(n=8)$.

All differentiated adenocarcinomas were tubular adenocarcinoma, and all undifferentiated adenocarcinomas were signet-ring cell carcinoma. In 48 patients with 48 lesions of $\mathrm{M}$ $\mathrm{NBI}$-DLLs, males predominated (M/ F, 27/21), with average age 66 years (range 41 to 81 ). Tumor locations were $U>L>M$ $=22 / 14 / 12$, respectively. Mean tumor diameter was $9.7 \mathrm{~mm}$ (range 1 to 43). For macroscopic type, more flat or depressed than elevated types of tumors were found (26/22, respectively). 
- Table 4 Helicobacter pylori infection status of M-NBI-DLLs.

\begin{tabular}{|c|c|c|c|c|c|}
\hline & $\begin{array}{l}\text { GA-FG } \\
n=25\end{array}$ & $\begin{array}{l}\text { GA-FGM } \\
n=1\end{array}$ & $\begin{array}{l}\text { Differentiated } \\
\text { adenocarcinom } \\
n=14\end{array}$ & $\begin{array}{l}\text { Undifferentiated } \\
\text { adenocarcinoma } \\
\mathrm{n}=8\end{array}$ & $\begin{array}{l}\text { Total } \\
n=48\end{array}$ \\
\hline Number of lesions evaluated for infection status & 24 & 1 & 14 & 8 & 47 \\
\hline H. pylori-positive & $4(16.7 \%)$ & 0 & $3(21.4 \%)$ & $1(12.5 \%)$ & $8(17.0 \%)$ \\
\hline H. pylori-negative & $20(83.3 \%)$ & $1(100 \%)$ & $11(78.6 \%)$ & $7(87.5 \%)$ & $39(82.9 \%)$ \\
\hline - H. pylori-naïve & 17 & 1 & 0 & 6 & 24 \\
\hline - Post-H. pylori eradication & 2 & 0 & 7 & 0 & 9 \\
\hline $\begin{array}{l}\text { - Suspected past infection (unidentified in eradication } \\
\text { history) }\end{array}$ & 1 & 0 & 4 & 1 & 6 \\
\hline
\end{tabular}

\section{Histological findings}

- Table 3 shows histological features of M-NBI-DLLs. The frequency of four distinct histological findings in M-NBI-DLLs were as follows. Well-differentiated adenocarcinoma with lowgrade atypia was seen in all differentiated adenocarcinoma lesions. Similarity of surface structure was seen in 10 of 14 differentiated adenocarcinoma lesions. Partially covered with a nonneoplastic mucosa was seen in six of 14 differentiated adenocarcinoma lesions. Completely covered with a non-neoplastic mucosa was seen in all GA-FG and undifferentiated adenocarcinoma lesions. In GA-FGM, a well-differentiated adenocarcinoma with low-grade atypia and partially covered with a non-neoplastic mucosa was found.

\section{$H p$ infection status}

Thirty-nine of $47(83.0 \%)$ patients with M-NBI-DLLs were $\mathrm{Hp}$ negative (GA-FG/GA-FGM/differentiated adenocarcinoma/undifferentiated adenocarcinoma; 20/1/11/7, respectively) ( $>$ Table4). Among $39 \mathrm{Hp}$-negative patients with M-NBI-DLLs, 24 were $\mathrm{Hp}$-naïve, and nine were post-eradication. Six of 39 patients with M-NBI-DLLs were $\mathrm{Hp}$-negative in clinical findings, although mucosal atrophy was present in endoscopic findings and past infection was suspected. However, $\mathrm{Hp}$ infection status in six patients with M-NBI-DLLs was difficult to discern because no eradication history could be found.

\section{Case presentations}

Representative cases of each histological type investigated in this study are shown in > Fig. 3, > Fig. 4, > Fig. 5 and > Fig. 6.

On a conventional endoscopy with white-light imaging (WLI), a whitish, submucosal tumor (SMT) shape of an elevated lesion about $6 \mathrm{~mm}$ in size was observed in the gastric fundus (GA-FG, Hp-naïve, > Fig.3). Dilated vessels with branch architecture were seen in the superficial layer of the lesion without atrophic changes in the surrounding background mucosa. M$\mathrm{NBI}$ revealed dilatations of the intervening part (IP) and crypt opening (CO), regular MS and MV pattern and no clear DL. Pathological findings showed a proliferation of neoplastic cells similar to fundic gland cells invading from the deep layer to beneath the superficial layer of the mucosa, with partial infiltration into the submucosal layer. Immunohistochemical staining revealed the lesion was positive for pepsinogen-I, $\mathrm{H}^{+} / \mathrm{K}^{+}$-ATPase and MUC6, and negative for MUC5AC, MUC2, and CD10, leading to a final pathological diagnosis of the lesion as $U$, adenocarcinoma of fundic-gland type, $0-I l a, 6 \times 5 \mathrm{~mm}$, pT1b/SM1 $(250 \mu \mathrm{m})$, pUL0, Ly0, V0, pHM0, and pVM0. The superficial layer of the tumor was completely covered with non-neoplastic epithelial cells, which may have contributed to the diagnosis of being non-cancerous by M-NBI examination.

On conventional endoscopy with WLI, a reddish, SMT shape of an elevated lesion about $10 \mathrm{~mm}$ in size was observed in the gastric fundus (GA-FGM, Hp-naïve, > Fig. 4). M-NBI revealed dilatations of the IP and CO, regular MS and MV pattern and no clear DL. Based on endoscopic findings, a reddish elevated type of GA-FG was suspected. Pathological findings showed proliferation of neoplastic cells, similar to fundic gland cells, invading from the middle layer to the deep layer of the mucosa, with partial infiltration into the submucosal layer $(700 \mu \mathrm{m})$. Immunohistochemical staining revealed that the lesion was diffusely positive for pepsinogen-I and MUC6, while sporadically positive for $\mathrm{H}^{+} / \mathrm{K}^{+}$-ATPase. However, MUC5AC-positive neoplastic cells differentiated into foveolar epithelium - like cells and were detected on the superficial layer of the mucosa. The final pathological diagnosis of the lesion was $U$, adenocarcinoma of fundic-gland mucosal type, 0-Ila, $15 \times 13 \mathrm{~mm}$, pT1b/SM2 (700 $\mu \mathrm{m})$, pUL0, Ly0, V0, pHM0, and pVM0. A diagnosis of a non-cancerous lesion by M-NBI was made based on the low irregularity of the tumor exposed on the superficial layer of the mucosa (differentiated adenocarcinoma with low-grade atypia), and the coexistence of and/or coverage with non-neoplastic epithelial cells over the lesion.

On a conventional endoscopy with WLI, a whitish, depressed lesion about $8 \mathrm{~mm}$ in size was observed on the greater curvature of the gastric antrum. M-NBI revealed regular MS and MV pattern and no clear DL (undifferentiated adenocarcinoma, $\mathrm{Hp}$ naïve, $>$ Fig.5). The final pathological diagnosis of the lesion was L, signet-ring cell carcinoma, $0-I l c, 7 \times 6 \mathrm{~mm}, \mathrm{pT} 1 \mathrm{a} / \mathrm{M}$, 

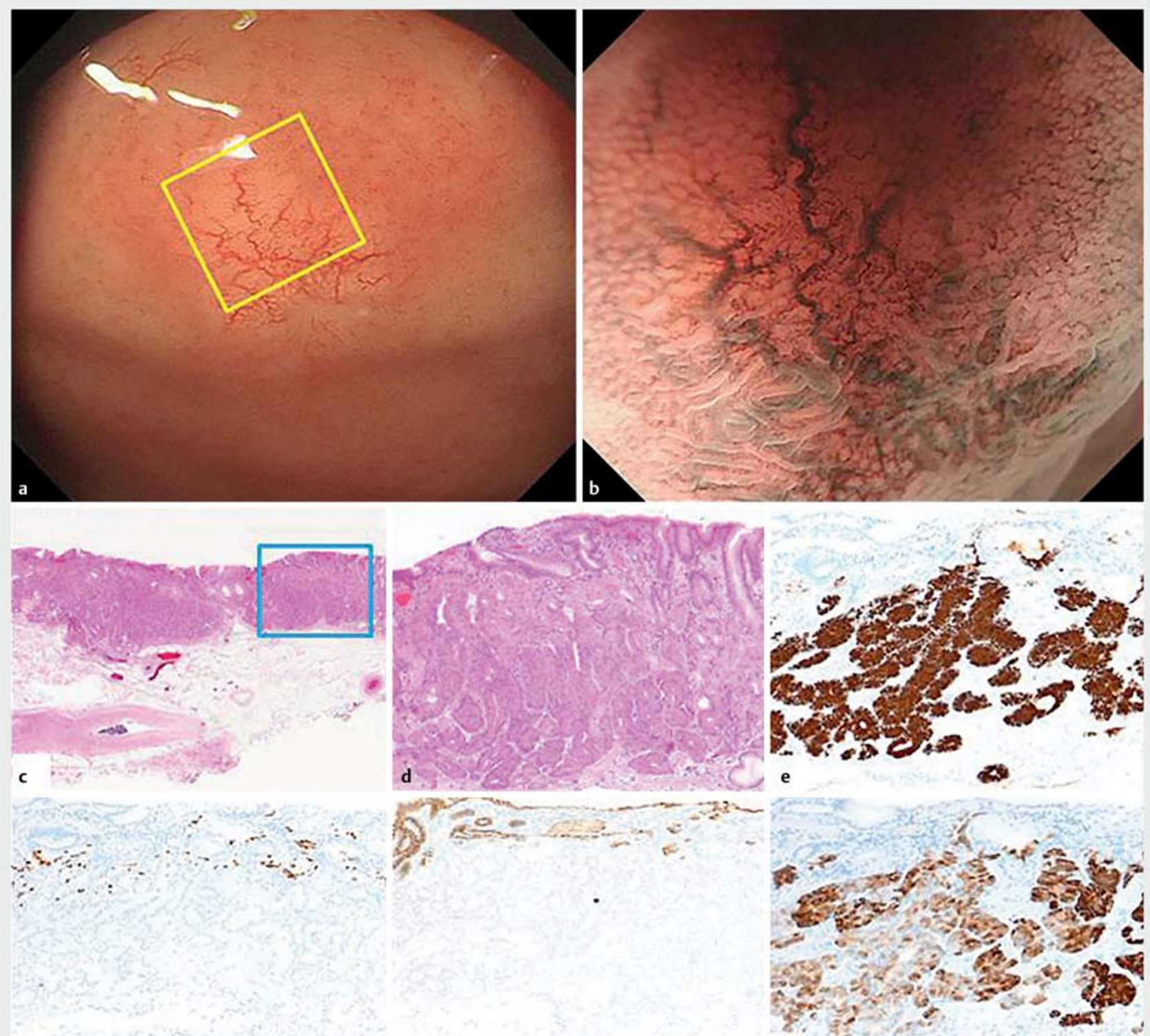

f

g

h

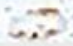

- Fig. 3 Endoscopic and histological findings of GA-FG. a WLI. A whitish, submucosal tumor in the shape of an elevated lesion, $6 \mathrm{~mm}$ in size, was detected in the gastric fornix. There were dilated vessels with branch architecture at the surface of the lesion. The yellow square area corresponds to $\mathbf{b}$. $\mathbf{b}$ Highest power magnification with NBI. M-NBI demonstrates dilatation of the CO and IP. There was no clear DL (MESDA-G: regular MV pattern plus regular MS pattern without a DL). c Histological findings. The blue square area corresponds to $\mathbf{d}$. $\mathbf{d}$ Pathological findings showed proliferation of neoplastic cells, similar to fundic gland cells, invading from the deep layer to beneath the surface of the mucosa. The superficial layer of tumor was completely covered with noncancerous epithelial cells. e, $\mathbf{f}, \mathbf{g}, \mathbf{h}$ Immunohistological findings. e pepsinogenI positive. $\mathbf{f} \mathrm{H}^{+} / \mathrm{K}^{+}$-ATPase partially positive. $\mathbf{g}$ MUC5AC negative. $\mathbf{h}$ MUC6 positive.

CO, crypt opening, DL, demarcation line; IP, intervening part; MESDA-G, magnifying endoscopy simple diagnostic algorithm for early gastric cancer; M-NBI, magnifying endoscopy with narrow-band imaging; MS, microsurface; MV, microvascular; NBI, narrow-band imaging; WLI, white-light imaging

pUL0, Ly0, V0, pHM0, and pVM0. Signet-ring cell carcinoma was confined to the middle layer of the mucosa. The superficial layer of the tumor was completely covered with a thick layer of non-neoplastic epithelial cells, which may have contributed to a diagnosis of a non-cancerous lesion by M-NBI.
On a conventional endoscopy with WLI, a pale-red depressed lesion about $10 \mathrm{~mm}$ in size with an obscure border was observed on the lesser curvature of the gastric antrum (differentiated adenocarcinoma, post-Hp eradication, - Fig. 6). M-NBI revealed regular MS and MV pattern and no clear DL. The final pathological diagnosis of the lesion was L, well-differentiated 

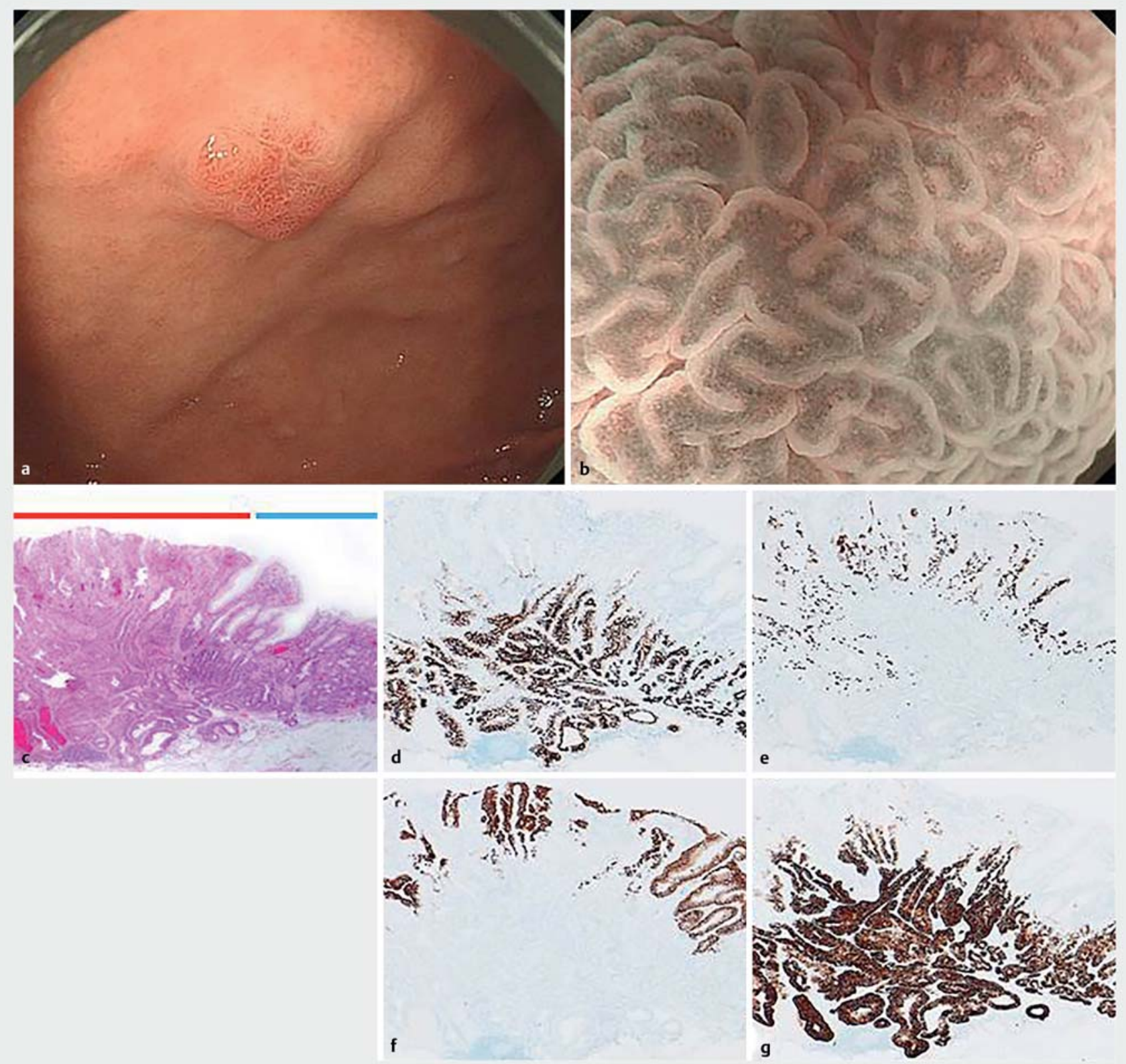

- Fig. 4 Endoscopic and histological findings of GA-FGM. a WLI. A reddish, submucosal tumor shaped as an elevated lesion, $10 \mathrm{~mm}$ in size, was detected in the gastric fornix. $\mathbf{b}$ Highest power magnification with NBI. There was no clear DL (MESDA-G: regular MV pattern plus regular MS pattern without a DL). c Histological findings. Atypia of the tumor exposed on the superficial layer of the lesion was extremely low (red bar), where coexistence of and/partial coverage with non-cancerous epithelial cells was observed (blue bar). $\mathbf{d}, \mathbf{e}, \mathbf{f}, \mathbf{g}$ Immunohistological findings. d pepsinogen-I positive. $\mathrm{H}^{+} / \mathrm{K}^{+}$-ATPase positive. $\mathbf{f}$ MUC5AC positive. $\mathbf{g}$ MUC6 positive.

$\mathrm{DL}$, demarcation line; GA-FGM, gastric adenocarcinoma of fundic-gland mucosal type; MS, microsurface; MV, microvascular; NBI, narrowband imaging; WLI, white-light imaging.

adenocarcinoma, $0-\| l c, 14 \times 6 \mathrm{~mm}, \mathrm{pT} 1 \mathrm{a} / \mathrm{M}$, pUL0, Ly0, V0, $\mathrm{pHMO}$, and pVMO. A diagnosis of a non-cancerous lesion by MNBI was made based on the presence of a differentiated adenocarcinoma with low-grade atypia and the partial coexistence of non-neoplastic epithelial cells. Moreover, the surface structure of the cancerous region resembled the non-cancerous region surrounding it, which may have made it more difficult to determine DL on endoscopic imaging.

\section{Discussion}

Diagnostic performance of M-NBI for EGC is very high, with Yao et al. reporting the accuracy, sensitivity and specificity of its diagnoses as $98.1,85.7$, and $99.4 \%$, respectively [24]. The NBI system is an optical image-enhanced technology containing a narrow-band filter with central wavelengths of 415 and $540 \mathrm{~nm}$. Because light with such wavelengths is absorbed by hemoglobin and propagates shallowly within the mucosal tissue, 


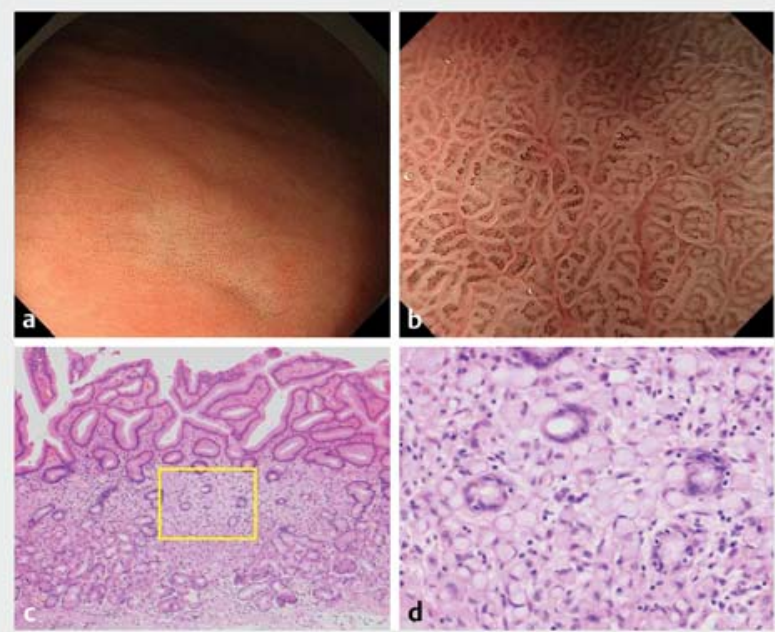

- Fig. 5 Endoscopic and histological findings of undifferentiated adenocarcinoma. a WLI. A whitish, depressed lesion, $8 \mathrm{~mm}$ in size, was detected in the greater curvature of the lower third of the stomach. b Highest power magnification with NBI. There was no clear DL (MESDA-G: regular MV pattern plus regular MS pattern without a DL). c Histological findings. The superficial mucosal layer was completely covered with a non-neoplastic mucosa. Signet-ring cell carcinoma was present only in the middle layer of the mucosa. $\mathbf{d}$ Magnified view of the yellow square area in (c). DL, demarcation line; MESDA-G, magnifying endoscopy simple diagnostic algorithm for early gastric cancer; MS, microsurface; MV, microvascular; NBI, narrow-band imaging; WLI, white-light imaging.

subepithelial MV architecture and mucosal MS are visualized in high contrast.

However, M-NBI is only suitable for visualizing MS and MV in the upper layer of the mucosa and unsuitable for visualizing lesions in deep layers of the mucosa [25]. Therefore, M-NBI is not suitable for visualizing lesions within or deeper than the deep layer of the mucosa and those with a superficial layer of nonneoplastic epithelial cells. Thus, such lesions can be considered as M-NBI-DLLs. Histological types presenting with the abovementioned histostructural findings include GA-FG, undifferentiated adenocarcinoma, and moderately differentiated adenocarcinoma [16, 26-28].

GA-FG accounted for the largest number of M-NBI-DLLs. In GA-FG, neoplastic cells, which originate from fundic glands and are similar to fundic gland cells, proliferate mainly in the deep layer of the mucosa. In all GA-FG cases, tumor was not exposed on the surface of the lesion.

Meanwhile, all undifferentiated adenocarcinomas of M-NBIDLLs were signet-ring cell carcinomas. Undifferentiated adenocarcinomas, especially signet-ring cell carcinomas, are thought to originate from the proliferative cell zone in the gastric gland neck and expand laterally within the middle layer of the mucosa during the initial stage of proliferation. During this stage, little destruction of the mucosal structure by proliferating cells occurs. All cases of signet-ring cell carcinoma were confined within the middle layer of the mucosa.
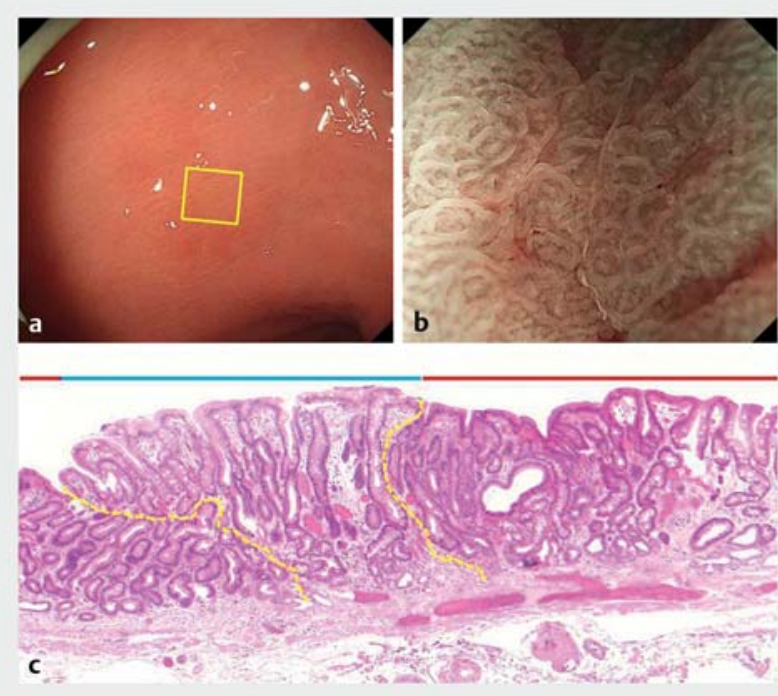

- Fig. 6 Endoscopic and histological findings of differentiated adenocarcinoma. a WLI. A reddish, depressed lesion was detected in the lesser curvature of the lower third of the stomach. The margin of the lesion was unclear. The yellow square area corresponds to $\mathbf{b}$. b Highest power magnification with NBI. There was no clear DL (MESDA-G: regular MV pattern plus regular MS pattern without a DL). c Histological findings. A well-differentiated adenocarcinoma with low-grade atypia (red bar) was present. Non-neoplastic mucosa was present (blue bar: the area between the two yellow, dashed lines). The surface structures of cancerous and non-neoplastic mucosa near the tumor are relatively similar.

DL, demarcation line; MESDA-G, magnifying endoscopy simple diagnostic algorithm for early gastric cancer; MS, microsurface; MV, microvascular; NBI, narrow-band imaging; WLI, white-light imaging.

Furthermore, several moderately differentiated adenocarcinomas have unique histostructural findings, in which neoplastic cells expand continuously from the portion exposed on the surface of the lesion. Beneath non-neoplastic epithelial cells, these neoplastic cells laterally invade the middle layer of the mucosa as the primary localization. Yao et al. [26] described a case in which determination of the horizontal margin with M-NBI was difficult. However, in this type of moderately differentiated adenocarcinoma, lesions are typically found with a portion exposed on the superficial layer, and it is not difficult to diagnose cancerous or non-cancerous; thus, such lesions were not considered difficult to diagnose in this study.

Based on these results, lesions with specific histostructural findings in which the tumor is not exposed on the surface of the lesion and the superficial layer is completely covered with non-neoplastic epithelial cells can be considered M-NBI-DLLs.

However, Okada et al. [29] reported that, regarding undifferentiated adenocarcinoma, including even signet-ring cell carcinoma confined to the middle layer of the mucosa, when foveolar epithelium is destroyed, the IP can be dilated and recognized by M-NBI. Furthermore, we have reported the following $\mathrm{M}-\mathrm{NBI}$ findings characteristic for GA-FG: absence of clear $\mathrm{DL}$, dilation of the IP, dilation of the $\mathrm{CO}$, and MV without dis- 
tinct irregularities [16]. All of these characteristics may be attributable to modification of non-neoplastic mucosa depending on the original locations of cancerous cells and forms of cellular proliferation and invasion. Such M-NBI findings, in which it may not be feasible to determine whether the lesion is cancer or non-cancerous, are nevertheless of value in providing necessary information suggestive of undifferentiated cancer and GA-FG.

In comparison, lesions considered M-NBI-DLLs, albeit exposed on the superficial layer, included GA-FGM and differentiated adenocarcinoma. In GA-FGM, the cancerous region is exposed on the superficial layer of the tumor. This can be detected as irregular MV/ MS patterns by M-NBI with such lesions more likely to be diagnosed as cancer $[16,17]$. In this study, two of three GA-FGM cases were diagnosed as cancer by M$\mathrm{NBI}$, while the remaining case was M-NBI-DLL and was diagnosed as non-cancerous. The pathological findings for this GAFGM misdiagnosed as non-cancerous were that a region of foveolar - epithelial cancer with extremely low-grade atypia was exposed on the superficial layer of the tumor that was partially covered with non-neoplastic epithelial cells. There is no established consensus on endoscopic features of GA-FGM due to the rarity of such cases. Cases exist that are difficult to diagnose, as represented by the above-mentioned GA-FGM case in which MNBI failed to judge its MS/ MV pattern as "regular" due to the extremely low grade of atypia, despite a part of the tumor being exposed on the superficial layer. Thus, further study is warranted.

In differentiated adenocarcinoma of M-NBI-DLLs, all lesions were cancer with extremely low-grade atypia. Furthermore, some lesions showed the similarity of surface structures and partially covered and/or mixed with a non-neoplastic mucosa.

Based on these results, we envisioned difficulties in making a cancer diagnosis by M-NBI, even for cases in which tumor was exposed on the superficial layer of the lesion, in the following specific situations. First, it is difficult to determine a DL along non-cancerous areas in lesions with extremely low atypia, such as differentiated adenocarcinoma with low-grade atypia in which surface structures are similar between cancerous and surrounding non-cancerous tissues. Second, it is also difficult to detect the presence of tumors when non-neoplastic epithelial cells coexist and/or partially cover the superficial layer of lesions.

In regard to $\mathrm{Hp}$ infection status, endoscopic demarcation and a qualitative diagnosis may be more difficult for post- $\mathrm{Hp}$ eradication gastric cancers since non-neoplastic epithelial cells may coexist and/or cover the superficial layer of the tumor and show surface differentiation with low cellular proliferation at the luminal surface [30-33]. Post-Hp eradication gastric cancers accounted for 77 out of 456 cases in this study. Nine of the $77(11.7 \%)$ post-Hp eradication gastric cancers were $\mathrm{M}$ NBI-DLLs. In terms of histological types, seven cases of differentiated adenocarcinoma and two cases of GA-FG were found. Post-Hp eradication gastric cancers accounted for half the total of 14 differentiated adenocarcinoma cases of M-NBI-DLLs. This suggested that $\mathrm{Hp}$ eradication is one factor complicating an $\mathrm{M}$ $\mathrm{NBI}$ diagnosis. Hp-naïve gastric cancer (24 lesions) and post-Hp eradication gastric cancer ( 9 lesions) collectively accounted for nearly $70 \%$ (33/48 lesions) of M-NBI-DLLs in this study. Together with cases for which the eradication history was unknown but were assumed to have a history of past $\mathrm{Hp}$ infection, 39 of $48 \mathrm{M}-\mathrm{NBI}$-DLLs (82.9\%) were $\mathrm{Hp}$-negative gastric cancers, demonstrating that the majority of M-NBI-DLLs were $\mathrm{Hp}$-negative gastric cancers.

As $\mathrm{Hp}$ eradication treatments become more widespread, the $\mathrm{Hp}$ infection rate has been drastically decreasing with time [34], and relative incidences of $\mathrm{Hp}$-negative gastric cancers are expected to increase in the future. Accordingly, the incidence of lesions that are difficult to diagnose on M-NBI may likely increase.

Limitations of the current study include its single-center nature, a retrospective design, a proportion bias of each histological types of gastric cancer based on the institution, a lack of analysis of inter - intra reliability, and investigation of false-positive cases (an endoscopic cancer diagnosis, but a non-cancerous pathological diagnosis).

\section{Conclusion}

In conclusion, this study revealed that M-NBI-DLLs were classified into four histological types as follows: GA-FG, GA-FGM, differentiated adenocarcinoma, and undifferentiated adenocarcinoma. The following histological features may be attributed to difficulties in diagnosis by M-NBI: (1) the superficial layer of a tumor completely covered with non-cancerous epithelial cells; (2) extremely low atypia of tumor (well-differentiated adenocarcinoma with low-grade atypia); (3) high similarity of surface structures between cancerous and surrounding non-cancerous tissues; and (4) the coexistence of and/or coverage with noncancerous epithelial cells over the superficial layer of the tumor. For accurate diagnosis of M-NBI-DLLs, it may be necessary to fully understand endoscopic features of these lesions by WLI and $\mathrm{M}-\mathrm{NBI}$ based on these histological characteristics and to take a biopsy precisely. However, lesions completely covered with non-neoplastic mucosa are unable to be visualized on $\mathrm{M}$ $\mathrm{NBI}$, and highlight a limitation of the diagnostic capacity of MNBI. Moreover, for lesions presenting with a histostructural finding of well-differentiated adenocarcinoma with low-grade atypia and/or high similarity of surface structure between cancerous and surrounding non-cancerous tissues, which can be visualized as tumors on $\mathrm{M}-\mathrm{NBI}$, changes in MS and/or MV patterns are minute, meaning that it can be difficult to judge irregularities. In future, further investigations will be needed to establish a new judgment criterion for determining the regularity or irregularity of such lesions and discovering a new characteristic of M-NBI findings that suggests cancer.

\section{Competing interests}

The authors declare that they have no conflict of interest. 


\section{References}

[1] Kaltenbach T, Sano Y, Friedland S et al. American Gastroenterological Association (AGA) Institute technology assessment on image-enhanced endoscopy. Gastroenterology 2008; 134: 327-340

[2] Muto M, Yao K, Kaise M et al. Magnifying endoscopy simple diagnostic algorithm for early gastric cancer (MESDA-G). Dig Endosc 2016; 28: 379-393

[3] Ezoe Y, Muto M, Horimatsu T et al. Magnifying narrow-band imaging versus magnifying white-light imaging for the differential diagnosis of gastric small depressive lesions: a prospective study. Gastrointest Endosc 2010; 71: 477-484

[4] Ezoe $\mathrm{Y}$, Muto $\mathrm{M}$, Uedo $\mathrm{N}$ et al. Magnifying narrowband imaging is more accurate than conventional white-light imaging in diagnosis of gastric mucosal cancer. Gastroenterology 2011; 141: 2017-2025. e2013

[5] Morita Y, Fujiwara S, Tanaka S et al. A case of small early gastric cancer that was successfully detected by narrow band imaging magnifying endoscopy. Dig Endosc 2011; 23: 89-91

[6] Nagahama T, Yao K, Maki S et al. Usefulness of magnifying endoscopy with narrow-band imaging for determining the horizontal extent of early gastric cancer when there is an unclear margin by chromoendoscopy (with video). Gastrointest Endosc 2011; 74: 1259-1267

[7] Miwa K, Doyama H, Ito R et al. Can magnifying endoscopy with narrow band imaging be useful for low grade adenomas in preoperative biopsy specimens? Gastric Cancer 2012; 15: 170-178

[8] Tsuji Y, Ohata K, Sekiguchi M et al. Magnifying endoscopy with narrow-band imaging helps determine the management of gastric adenomas. Gastric Cancer 2012; 15: 414-418

[9] Maki S, Yao K, Nagahama T et al. Magnifying endoscopy with narrowband imaging is useful in the differential diagnosis between lowgrade adenoma and early cancer of superficial elevated gastric lesions. Gastric Cancer 2013; 16: 140-146

[10] Yao K, Nagahama T, Matsui T et al. Detection and characterization of early gastric cancer for curative endoscopic submucosal dissection. Dig Endosc 2013; 25: 44-54

[11] Hwang JW, Bae YS, Kang MS et al. Predicting pre- and post-resectional histologic discrepancies in gastric low-grade dysplasia: A comparison of white-light and magnifying endoscopy. J Gastroenterol Hepatol 2016; 31: 394-402

[12] Nonaka T, Inamori M, Honda $Y$ et al. Can magnifying endoscopy with narrow-band imaging discriminate between carcinomas and low grade adenomas in gastric superficial elevated lesions? Endosc Int Open 2016; 4: E1203-e1210

[13] Yoshimizu S, Yamamoto Y, Horiuchi Y et al. Diagnostic performance of routine esophagogastroduodenoscopy using magnifying endoscope with narrow-band imaging for gastric cancer. Dig Endosc 2018; 30: $71-78$

[14] Ueyama H, Yao T, Nakashima Y et al. Gastric adenocarcinoma of fundic gland type (chief cell predominant type): proposal for a new entity of gastric adenocarcinoma. Am J Surg Pathol 2010; 34: 609-619

[15] WHO Classification of Tumours Editorial Board. WHO Classification of Tumours. 5th ed, Vol 1 Digestive System tumours. Lyon: IARC; 2019

[16] Ueyama H, Yao T, Matsumoto K et al. Establishment of endoscopic diagnosis for gastric adenocarcinoma of fundic gland type (chief cell predominant type) usingmagnifying endoscopy with narrow-band imaging.50 Tokyo: Stomach Intestine; 2015: 1533-1547
[17] Fujiwara S, Yao K, Imamura K et al. M-NBI findings of gastric adenocarcinoma offundic gland type and adenocarcinoma with differentiation towards the fundic mucosa.50 Tokyo: Stomach Intestine; 2015: 1548-1558

[18] Ueyama H, Yao T, Nagahara A et al. Gastric Adenocarcinoma of fundic gland type.53 Tokyo: Stomach Intestine; 2018: 753-767

[19] Yoshimura D, Yoshimura R, Kato $S$ et al. Characteristics of gastric cancer without Helicobacter pylori Infection and its relation to background mucosa.53 Tokyo: Stomach Intestine; 2018: 658-670

[20] Yao K, Anagnostopoulos GK, Ragunath K. Magnifying endoscopy for diagnosing and delineating early gastric cancer. Endoscopy 2009; 41: 462-467

[21] Kimura K. An endoscopic recognition of the atrophic border and its significance in chronic gastritis. Endoscopy 1969; 3: 87-97

[22] Dixon MF. Gastrointestinal epithelial neoplasia: Vienna revisited. Gut 2002; 51: 130-131

[23] Nihon Igan G. Igan toriatsukai kiyaku. Tokyo: Kaneharashuppan; 2017

[24] Yao K, Doyama H, Gotoda T et al. Diagnostic performance and limitations of magnifying narrow-band imaging in screening endoscopy of early gastric cancer: a prospective multicenter feasibility study. Gastric Cancer 2014; 17: 669-679

[25] Fujisaki J, Horiuchi Y, Yamamoto $\mathrm{N}$ et al. Characteristic findings of difficult cases to diagnose using ME-NBI. Stomach Intestine (Tokyo) 2015; 50: 279-288

[26] Yao K, Nagahama T, Maki S et al. Usefulness of magnifying endoscopy with Narrow-band imaging for determining the margins of superficial flat Type (0 Ilb) of early gastric carcinomas. Stomach Intestine (Tokyo) 2010; 45: 86-100

[27] Yao K, Fujiwara S, Nagahama T et al. Diagnostic performance and limitations ofmagnifying narrow-band imaging for the diagnosis of minute gastric cancer. Stomach Intestine (Tokyo) 2013; 48: 843-856

[28] Nagahama T, Imamura K, Kojima T et al. Usefulness and limitations of the magnifying endoscopy with narrow band imaging for delineation of very well diff erentiated adenocarcinoma of the stomach. Stomach Intestine (Tokyo) 2015; 50: 267-278

[29] Okada K, Fujisaki ], Kasuga A et al. Diagnosis of undifferentiated type early gastric cancers by magnification endoscopy with narrow-band imaging. J Gastroenterol Hepatol 2011; 26: 1262-1269

[30] Ito M, Tanaka S, Takata S et al. Morphological changes in human gastric tumours after eradication therapy of Helicobacter pylori in a short-term follow-up. Aliment Pharmacol Ther 2005; 21: 559-566

[31] Kobayashi M, Hashimoto S, Nishikura K et al. Magnifying narrow-band imaging of surface maturation in early differentiated-type gastric cancers after Helicobacter pylori eradication. J Gastroenterol 2013; 48: $1332-1342$

[32] Kitamura Y, Ito M, Matsuo T et al. Characteristic epithelium with lowgrade atypia appears on the surface of gastric cancer after successful Helicobacter pylori eradication therapy. Helicobacter 2014; 19: 289295

[33] Saka A, Yagi K, Nimura S. Endoscopic and histological features of gastric cancers after successful Helicobacter pylori eradication therapy. Gastric Cancer 2016; 19: 524-530

[34] Kamada T, Haruma K, Ito M et al. Time trends in Helicobacter pylori infection and atrophic gastritis over 40 years in Japan. Helicobacter 2015; 20: 192-198 